BULLETIN (New Series) OF THE

AMERICAN MATHEMATICAL SOCIETY

Volume 37, Number 1, Pages 39-44

S 0273-0979(99)00805-8

Article electronically published on December 21, 1999

\title{
ELEMENTARY DERIVATION OF THE EQUIVALENCE OF MASS AND ENERGY
}

\author{
ALBERT EINSTEIN
}

The special theory of relativity grew out of the Maxwell electromagnetic equations. So it came about that even in the derivation of the mechanical concepts and their relations the consideration of those of the electromagnetic field has played an essential role. The question as to the independence of those relations is a natural one because the Lorentz transformation, the real basis of the special relativity theory, in itself has nothing to do with the Maxwell theory and because we do not know the extent to which the energy concepts of the Maxwell theory can be maintained in the face of the data of molecular physics. In the following considerations, except for the Lorentz transformation, we will depend only on the assumption of the conservation principles for impulse and energy.

We begin by making plausible the expressions for impulse and energy of the material particle in the well known way. The fundamental invariant of the Lorentz transformation is

$$
d s^{2}=d t^{2}-d x^{2}-d y^{2}-d z^{2},
$$

or

$$
d s=d t\left(1-u^{2}\right)^{1 / 2},
$$

where

$$
u^{2}=\left(\frac{d x}{d t}\right)^{2}+\left(\frac{d y}{d t}\right)^{2}+\left(\frac{d z}{d t}\right)^{2}=u_{1}^{2}+u_{2}^{2}+u_{3}^{2} .
$$

If one divides the components of the contravariant vector $(d t, d x, d y, d z)$ by $d s$, one obtains the vector

$$
\left(\frac{1}{\left(1-u^{2}\right)^{1 / 2}}, \frac{u_{1}}{\left(1-u^{2}\right)^{1 / 2}}, \frac{u_{2}}{\left(1-u^{2}\right)^{1 / 2}}, \frac{u_{3}}{\left(1-u^{2}\right)^{1 / 2}}\right) .
$$

Let $(d t, d x, d y, d z)$ belong to the world-line of a material particle of mass $m$. We obtain a vector connected with the motion of the latter by multiplying by $m$ the four-vector of velocity that we have just written down. We thus have

$$
\left(\eta^{\sigma}\right)=\left(\frac{m}{\left(1-u^{2}\right)^{1 / 2}}, \frac{m u_{i}}{\left(1-u^{2}\right)^{1 / 2}}\right)
$$

where the index $i$ runs from 1 to 3 .

2000 Mathematics Subject Classification. Primary 83-02, 83A05.

Reprinted from Bull. Amer. Math. Soc. 41 (1935), 223-230.

The Eleventh Josia Willard Gibbs Lecture, delivered at Pittsburgh, December, 28, 1934, under the auspices of this Society, at a joint meeting of this Society, the American Physical Society, and Section A of the A.A.A.S. 
Neglecting the third power of the velocity, we can express the components of this vector by

$$
\left(\eta^{\sigma}\right)=\left(m+\frac{1}{2} m u^{2}, m u_{i}\right)
$$

The space components of $\left(\eta^{\sigma}\right)$ express in this approximation the components of the impulse in classical mechanics, while the time component, aside from the additive constant $m$, expresses the kinetic energy of the material point.

If one goes back to the exact expression for $\left(\eta^{\sigma}\right)$, it is therefore natural to take

$$
\frac{m u_{i}}{\left(1-u^{2}\right)^{1 / 2}}
$$

as the impulse and

$$
m\left(\frac{1}{\left(1-u^{2}\right)^{1 / 2}}-1\right)
$$

as the kinetic energy of the particle. However, how is one to interpret the time component $m /\left(1-u^{2}\right)^{1 / 2}$ itself, the expression for which after all is the really significant one? Here it is natural to give it directly the meaning of energy, hence to ascribe to the mass-point in a state of rest the rest-energy $m$ (with the usual time unit, $\left.m c^{2}\right)$.

Of course, this derivation cannot pretend to be a proof since in no way is it shown that this impulse satisfies the impulse-principle and this energy the energyprinciple if several particles of the same kind interact with one another; it may be a priori conceivable that in these conservation-principles different expressions of the velocity are involved.

Furthermore, it is not perfectly clear as to what is meant in speaking of the restenergy, as the energy is defined only to within an undetermined additive constant; in connection with this, however, the following is to be remarked. Every system can be looked upon as a material point as long as we consider no processes other than changes in its translation velocity as a whole. It has a clear meaning, however, to consider changes in the rest-energy in case changes are to be considered other than mere changes in translation velocity. The above interpretation asserts, then, that in such a transformation of a material point its inertial mass changes as the rest-energy; this assertion naturally requires a proof.

What we will now show is the following. If the principles of conservation of impulse and energy are to hold for all coordinate systems which are connected with one another by the Lorentz transformations, then impulse and energy are really given by the above expressions and the presumed equivalence of mass and rest-energy also exists.

We start from some simple kinematic consequences of the Lorentz transformation:

$$
t=\frac{t^{\prime}+v x^{\prime}}{\left(1-v^{2}\right)^{1 / 2}}, \quad x=\frac{x^{\prime}+v t^{\prime}}{\left(1-v^{2}\right)^{1 / 2}}, \quad y=y^{\prime}, \quad z=z^{\prime},
$$

where $v$ is the relative velocity of the coordinate systems $K$ and $K^{\prime}$. The same relations hold for the differentials $d x$, etc. By suitable division one obtains the law of transformation of the velocities:

$$
u_{1}=\frac{u_{1}^{\prime}+v}{1+u_{1}^{\prime} v}, \quad u_{2}=\frac{u_{2}^{\prime}\left(1-v^{2}\right)^{1 / 2}}{1+u_{1}^{\prime} v}, \quad u_{3}=\frac{u_{3}^{\prime}\left(1-v^{2}\right)^{1 / 2}}{1+u_{1}^{\prime} v} .
$$


From this one obtains

$$
u^{2}=\frac{u^{\prime 2}+2 u_{1}^{\prime} v+v^{2}-u_{2}^{\prime 2} v^{2}-u_{3}^{\prime 2} v^{2}}{\left(1+u_{1}^{\prime} v\right)^{2}},
$$

and

$$
\frac{1}{\left(1-u^{2}\right)^{1 / 2}}=\frac{1+u_{1}^{\prime} v}{\left(1-u^{\prime 2}\right)^{1 / 2}\left(1-v^{2}\right)^{1 / 2}},
$$

as well as

$$
\begin{aligned}
& \frac{u_{1}}{\left(1-u^{2}\right)^{1 / 2}}=\frac{u_{1}^{\prime}+v}{\left(1-u^{\prime 2}\right)^{1 / 2}\left(1-v^{2}\right)^{1 / 2}}, \\
& \frac{u_{2}}{\left(1-u^{2}\right)^{1 / 2}}=\frac{u_{2}^{\prime}}{\left(1-u^{\prime 2}\right)^{1 / 2}}, \quad \frac{u_{3}}{\left(1-u^{2}\right)^{1 / 2}}=\frac{u_{3}^{\prime}}{\left(1-u^{\prime 2}\right)^{1 / 2}} .
\end{aligned}
$$

We introduce now for the following concept of the particle-pair. By this we understand two material points with equal and opposite velocities referred to $K^{\prime}$ (and later to be chosen of equal mass). The two particles are designated by the indices + or - . Hence $u_{+}^{\prime}=u_{-}^{\prime}, u_{1+}^{\prime}=-u_{1-}^{\prime}$, etc. Applying to these our transformation equations after addition, we get

$$
\begin{aligned}
& \frac{1}{\left(1-u_{+}^{2}\right)^{1 / 2}}+\frac{1}{\left(1-u_{-}^{2}\right)^{1 / 2}}=\frac{2}{\left(1-u^{\prime 2}\right)^{1 / 2}\left(1-v^{2}\right)^{1 / 2}}, \\
& \frac{u_{1+}}{\left(1-u_{+}^{2}\right)^{1 / 2}}+\frac{u_{1-}}{\left(1-u_{-}^{2}\right)^{1 / 2}}=\frac{2 v}{\left(1-u^{\prime 2}\right)^{1 / 2}\left(1-v^{2}\right)^{1 / 2}}, \\
& \frac{u_{2+}}{\left(1-u_{+}^{2}\right)^{1 / 2}}+\frac{u_{2-}}{\left(1-u_{-}^{2}\right)^{1 / 2}}=0, \\
& \frac{u_{3+}}{\left(1-u_{+}^{2}\right)^{1 / 2}}+\frac{u_{3-}}{\left(1-u_{-}^{2}\right)^{1 / 2}}=0 .
\end{aligned}
$$

The sums on the left sides of these equations depend, therefore, only on the velocity $u^{\prime}$ of the pair referred to the special system $K^{\prime}$ and on the relative velocity $v$ of $K^{\prime}$ with respect to $K$, not however on the direction in which the particles are moving.

We remark that the equations (1) can be derived more clearly if one considers directly the transformation for the sum of the four-vectors of the velocities of a particle-pair. I have chosen the above representation, however, because the conservation laws indicate the use of this 3-dimensionally inhomogeneous manner of writing.

We now go to the actual considerations. We assume that impulse and energy of a material point are given by expressions of the form

$$
I_{\nu}=m u_{\nu} F(u), \quad E=E_{0}+m G(u), \quad(\nu=1,2,3),
$$

where $F$ and $G$ are universal, even functions of the velocity $u$, which vanish for $u=0$. Then $m G(u)$ is the kinetic energy, $E_{0}$ the rest-energy of the material point, $m$ the rest-mass or, simply, the mass. It is here assumed that impulse and energy of the mass-point are independent of direction of motion and of the orientation of the mass-point relative to the velocity. It is further assumed that in impulse and energy the same mass-constant $m$ occurs, for which, however, we shall find later a partial justification.

We consider now the elastic eccentric collision between two particles of equal mass. One can always choose the coordinate-system $K^{\prime}$ so that, referred to the latter, the velocities of the masses before the collision are equal in magnitude and 
opposite in direction; how are the velocities after the collision constituted with reference to $K^{\prime}$ ? If the velocities after the collision were not likewise equal and opposite, the impulse law would be violated. If the common velocity of both masses after the collision were not equal in magnitude to that before the collision, in case the collision is elastic, the energy law would be violated. This holds independently of the particular law of dependence of impulse and energy on the velocity. The collision, therefore, changes only the direction of motion of the two mass-points referred to $K^{\prime}$. We can express this briefly as follows. A particle-pair before the collision is transformed by the latter again into a particle-pair of the same velocity $u^{\prime}$.

The right side of (1) is therefore not changed by the collision. It follows then, from (1), that, referred to $K$, we have for the states before and after the collision the equations:

$$
\begin{aligned}
& \frac{1}{\left(1-u_{+}^{2}\right)^{1 / 2}}+\frac{1}{\left(1-u_{-}^{2}\right)^{1 / 2}}=\frac{1}{\left(1-\bar{u}_{+}^{2}\right)^{1 / 2}}+\frac{1}{\left(1-\bar{u}_{-}^{2}\right)^{1 / 2}}, \\
& \frac{u_{i+}}{\left(1-u_{+}^{2}\right)^{1 / 2}}+\frac{u_{i-}}{\left(1-u_{-}^{2}\right)^{1 / 2}}=\frac{\bar{u}_{i+}}{\left(1-\bar{u}_{+}^{2}\right)^{1 / 2}}+\frac{\bar{u}_{i-}}{\left(1-\bar{u}_{-}^{2}\right)^{1 / 2}} .
\end{aligned}
$$

Barred quantities are those which refer to the state after the collision. These equations, which are valid in general for elastic collisions of equal masses, have the form of conservation equations; it may therefore be taken for granted that no other symmetrical or anti-symmetrical functions of the velocity-components exist which in the present case of the elastic collision of two identically constituted material points give anything analogous. Consequently we shall have to regard

$$
\frac{m u_{i}}{\left(1-u^{2}\right)^{1 / 2}}
$$

as impulse and

$$
m\left(\frac{1}{\left(1-u^{2}\right)^{1 / 2}}-1\right)
$$

as the kinetic energy of a particle. ${ }^{1}$

We now turn to the proof of the assertion that the mass is equal to the restenergy. For the total energy $E$ of a moving particle we shall have to take

$$
E=E_{0}+m\left(\frac{1}{\left(1-u^{2}\right)^{1 / 2}}-1\right)
$$

where we imagine that $E_{0}$ (rest-energy) and $m$ can be changed in the case of interactions of material points that are not elastic.

We now consider the inelastic collision between two particles of equal mass and equal rest-energy, which before the collision again form a particle-pair with respect to $K^{\prime}$ (velocities equal and opposite). We assume here for simplicity that the internal changes which the material points suffer in the collision are equal to each other. From the impulse principle referred to $K^{\prime}$ it follows as above that the final velocities of the two particles must be equal and opposite $\left(\bar{u}_{+}^{\prime}=-\bar{u}_{-}^{\prime}\right)$. The energy

\footnotetext{
${ }^{1}$ This must naturally vanish for $u=0$; for it is defined as the expenditure of energy necessary to impart the velocity $u$ to the particle initially at rest (without internal change).
} 
law gives then, referred to $K^{\prime}$ and $K$, respectively,

$$
\begin{aligned}
& 2 E_{0}+2 m\left(\frac{1}{\left(1-u^{\prime 2}\right)^{1 / 2}}-1\right)=2 \bar{E}_{0}+2 \bar{m}\left(\frac{1}{\left(1-\bar{u}^{\prime 2}\right)^{1 / 2}}-1\right), \\
& 2 E_{0}+m\left(\frac{1}{\left(1-u_{+}^{2}\right)^{1 / 2}}-1\right)+m\left(\frac{1}{\left(1-u_{-}^{2}\right)^{1 / 2}}-1\right) \\
& =2 \bar{E}_{0}+\bar{m}\left(\frac{1}{\left(1-\bar{u}_{+}^{2}\right)^{1 / 2}}-1\right)+\bar{m}\left(\frac{1}{\left(1-\bar{u}_{-}^{2}\right)^{1 / 2}}-1\right) .
\end{aligned}
$$

As the points form a pair before and after the collision, one can write the last equation on the basis of (1) in the form

$$
\begin{aligned}
E_{0}-m+ & \frac{m}{\left(1-u^{\prime 2}\right)^{1 / 2}\left(1-v^{2}\right)^{1 / 2}} \\
& =\bar{E}_{0}-\bar{m}+\frac{\bar{m}}{\left(1-\bar{u}^{\prime 2}\right)^{1 / 2}\left(1-v^{2}\right)^{1 / 2}} .
\end{aligned}
$$

The first equation we write analogously in the form

$$
E_{0}-m+\frac{m}{\left(1-u^{\prime 2}\right)^{1 / 2}}=\bar{E}_{0}-\bar{m}+\frac{\bar{m}}{\left(1-\bar{u}^{\prime 2}\right)^{1 / 2}} .
$$

Multiplying the last equation by $1 /\left(1-v^{2}\right)^{1 / 2}$ and subtracting from the previous one we get

or

$$
\left[\left(\bar{E}_{0}-E_{0}\right)-(\bar{m}-m)\right]\left(\frac{1}{\left(1-v^{2}\right)^{1 / 2}}-1\right)=0,
$$

$$
\bar{E}_{0}-E_{0}=\bar{m}-m \text {. }
$$

The rest-energy changes, therefore, in an inelastic collision (additively) like the mass. As the former, from the nature of the concept, is determined only to within an additive constant, one can stipulate that $E_{0}$ should vanish together with $m$. Then we have simply

$$
E_{0}=m,
$$

which states the principle of equivalence of inertial mass and rest-energy.

If we apply the impulse law to the $x$-component, it follows (for an inelastic collision) that

$$
m \frac{u_{+1}}{\left(1-u_{+}^{2}\right)^{1 / 2}}+m \frac{u_{-1}}{\left(1-u_{-}^{2}\right)^{1 / 2}}=\bar{m} \frac{\bar{u}_{+1}}{\left(1-\bar{u}_{+}^{2}\right)^{1 / 2}}+\bar{m} \frac{\bar{u}_{-1}}{\left(1-\bar{u}_{-}^{2}\right)^{1 / 2}},
$$

or by the application of the second of equations (1), for the state before and after the collision,

$$
\frac{m}{\left(1-u^{2}\right)^{1 / 2}}=\frac{\bar{m}}{\left(1-\bar{u}^{2}\right)^{1 / 2}} .
$$

The same relation follows also from equations (5) and (6) which were obtained from the energy principle. If, from the beginning, we had provided the expression for the impulse with a mass-constant different from that of the energy, these considerations would show that the impulse-mass changes in an inelastic collision like the energy-mass. This is a partial justification for setting both mass-constants equal to each other. 
The result of this consideration is therefore as follows. If for collisions of material points the conservation laws are to hold for an arbitrary (Lorentz) coordinatesystem, the well known expressions for impulse and energy follow, as well as the validity of the principle of equivalence of mass and rest-energy.

Professor G. D. Birkhoff has called my attention to the fact that in the book which he has written in collaboration with Professor R. E. Langer, Relativity and Modern Physics, quite similar considerations are given concerning collisions of particles, as well as concerning impulse and energy. In spite of this, I believe that the present derivations merit a certain amount of interest.

Thus, in the book just mentioned, essential use is made of the concept of force, which in the relativity theory has no such direct significance as it has in classical mechanics. This is connected with the fact that, in the latter, the force is to be considered as a given function of the coordinates of all the particles, which is obviously not possible in the relativity theory. Therefore I have avoided introducing the force concept.

Furthermore, I was concerned with avoiding making any assumption concerning the transformation character of impulse and energy with respect to a Lorentz transformation.

Institute FOR AdVAnCEd Study 